\title{
BMJ Open Identifying factors affecting latent tuberculosis treatment acceptance among healthcare workers: a retrospective analysis in a tertiary care centre
}

Maskit Bar-Meir (D) ,1,2 Gali Pariente, ${ }^{1}$ Ayal Romem, ${ }^{1,2}$ Yonit Wiener-Well ${ }^{2,3}$

To cite: Bar-Meir M, Pariente G, Romem A, et al. Identifying factors affecting latent tuberculosis treatment acceptance among healthcare workers: a retrospective analysis in a tertiary care centre. BMJ Open 2021;11:e047444. doi:10.1136/ bmjopen-2020-047444

- Prepublication history and additional supplemental material for this paper are available online. To view these files, please visit the journal online (http://dx.doi.org/10.1136/ bmjopen-2020-047444).

Received 28 November 2020 Accepted 24 August 2021
Check for updates

(c) Author(s) (or their employer(s)) 2021. Re-use permitted under CC BY-NC. No commercial re-use. See rights and permissions. Published by BMJ.

${ }^{1}$ Pediatric Infectious Diseases, Shaare-Zedek Medical Center, Jerusalem, Israel

${ }^{2}$ Faculty of Medicine, Hebrew University of Jerusalem, Jerusalem, Israel

${ }^{3}$ Infectious Diseases Unit, Shaare-Zedek Medical Center, Jerusalem, Israel

Correspondence to Dr Maskit Bar-Meir; mbarmeir@gmail.com

\section{ABSTRACT}

Objective Official guidelines recommend tuberculosis (TB) screening programmes for all healthcare workers (HCWs), along with offering treatment when latent TB infection (LTBI) is diagnosed. However, adherence to treatment among HCWs is lower compared with non-HCWs. The aim of the present study was to examine the rate of LTBI treatment acceptance among HCWs and to characterise the factors associated with non-acceptance.

Design and setting This was a retrospective cohort study. All HCWs diagnosed with LTBI, who had tuberculin skin test (TST) conversion during their work, between 2000 and 2015, in a single tertiary academic medical centre, and who consented to answer a questionnaire, were enrolled.

Results Overall, 147 of $219(67 \%)$ with TST conversion agreed to participate. Acceptance rate for LTBI treatment was only $16 \%$. The overall completion rate among those who accepted treatment was $87 \%$. HCWs' recall of discussing the importance of LTBI treatment with their caregiver had the strongest association with LTBI treatment acceptance: 23 of 52 HCWs (44\%) who recalled this discussion accepted treatment (adjusted $\mathrm{OR}=10.2$, $95 \% \mathrm{Cl}: 2.2$ to $47.6, p=0.003)$. Knowing the risk of developing TB was associated with 3.7 increased odds to accept treatment $(95 \% \mathrm{Cl}: 1.2$ to $11.8, \mathrm{p}=0.02)$. Conclusions LTBI acceptance rate was very low among our HCWs. Focusing on educating HCWs is potentially the key step towards an increased rate of LTBI treatment acceptance.

\section{INTRODUCTION}

Tuberculosis (TB) continues to be associated with significant disease burden around the world..$^{1}$ According to the Centers for Disease Control and Prevention (CDC), in 2018, about a quarter of the world's population had latent TB infection (LTBI), approximately 10.4 million people worldwide developed TB disease and 1.7 million died of TB. In the year 2018 in the USA, 9025 new TB cases were reported (2.8 cases per 100000 persons). ${ }^{2}$ In Israel, during 2014, 366 new TB cases were reported, and $\mathrm{TB}$ incidence was 4.4 cases per 100000 persons. ${ }^{3}$ BCG was administered

\section{Strengths and limitations of this study}

- This is a retrospective, interview-based study of healthcare workers who had tuberculin skin test conversion during their work, between 2000 and 2015 , in a single tertiary academic medical centre.

- The study examined the acceptance rate for latent tuberculosis infection treatment and analysed the main reasons for non-acceptance.

- Since it was an interview-based study, recall bias and reporting bias may have affected the participants' answers.

to 12-year-old children in Israel up to 1982. Since then, it is given only to selected highrisk groups.

The cumulative risk of LTBI reactivation is $5 \%-10 \%$, with the majority of cases occurring in the first 5 years following exposure. ${ }^{4}$ Several treatment options are available to reduce the risk of progression from LTBI to active disease. Isoniazid (INH), administered daily for 9 months, was the mainstay of therapy for decades, with reported efficacy rates ranging from $60 \%$ to $90 \%$, but with adherence rates of $44 \%-53 \% .^{5}$ However, shorter regimens such as 4 months of rifampicin (RIF) or 3 months of rifapentine plus INH resulted in higher completion rates and lower rates of discontinuation due to side effects. ${ }^{67}$

The risk of TB among healthcare workers (HCWs) is consistently higher than the risk among the general population worldwide. ${ }^{8}$ The CDC published guidelines for preventing the transmission of Mycobacterium tuberculosis in healthcare facilities and recommended TB screening programmes for all HCWs, along with offering treatment when LTBI is diagnosed. ${ }^{9}$ However, adherence to treatment in HCWs is lower compared with nonHCWs. ${ }^{5}$ A recent study found that only $39 \%$ of employees diagnosed with LTBI accepted treatment, ${ }^{6}$ however the reasons for not 
accepting treatment were not reported. The aim of the present study was to examine the rate of LTBI treatment acceptance among HCWs and to characterise the factors associated with non-acceptance.

\section{METHODS}

Shaare-Zedek Medical Center (SZMC), is a 1000-bed tertiary care academic hospital in Jerusalem, Israel, with approximately 3500 employees. Patients with suspected or proven TB infection are promptly placed in airborne infection isolation rooms, which are available throughout the building-in the internal medicine and paediatrics wards, in the emergency department and in the intensive care units.

TB screening for all SZMC HCWs is performed by a two-step tuberculin skin testing (TST), using five tuberculin units of purified protein derivative (PPD, Mantoux) (Tubersol, Sanofi Pasteur, Canada) injected intradermally and read within $48-72$ hours by trained nurses. A TST result of $\geq 10 \mathrm{~mm}$ in the transverse diameter is considered positive. TB screening is performed upon application for work in the hospital, and afterwards according to the risk status and known exposures. The highest risk includes HCWs in the emergency room, intensive care unit, pulmonary unit, radiology department and departments in which $\geq 3$ patients with TB per year were hospitalised. These HCWs were screened annually.

HCWs were diagnosed with LTBI if they had a negative first TST (upon acceptance for work or afterwards), followed by a positive one, with no time limitation between tests, and a chest radiograph with no signs of active TB. Interferon gamma release assay (IGRA) testing was not readily available during these years and was performed only upon request (of HCW or his caregiver). Two treatment options were offered: INH for 9 months or RIF for 4 months. Treatment is not mandatory and HCWs may choose to defer treatment.

All HCWs diagnosed with LTBI (eg, conversion of TST from negative to positive), while employed in the hospital, between 2000 and 2015, were identified via human resources and approached to participate in the study. HCWs who gave oral informed consent to participate were enrolled.

All participants were asked to answer a short telephone questionnaire (see online supplemental appendix) to assess their acceptance and completion of treatment for LTBI, as well as reasons for non-acceptance and noncompletion. Treatment acceptance was defined as taking the drugs recommended, for any period of time. Treatment completion was defined as fulfilling the allotted time recommended for therapy. HCWs answered questions regarding symptoms attributed to LTBI treatment, signs and symptoms that can be attributed to active TB disease, their health status, comorbidities and risk factors for TB disease. HCWs who did not accept LTBI treatment were asked for reasons for non-acceptance. Additionally, HCWs were asked whether they had a discussion with their caregiver regarding the importance of LTBI treatment, whether they had TB exposure (known exposure to patient with active $\mathrm{TB}$, or a visit to an endemic area) and whether they know the risk of LTBI developing into active disease (answers ranging between 5\% and 10\% were regarded correct). We postulated that such knowledge may affect the odds of treatment acceptance.

\section{Patient and public involvement}

Patients and the public were not involved in the design or performance of the study.

\section{Data analysis}

The primary outcome was the treatment acceptance rate. Analysis was performed with SPSS software V.25. Data are presented as mean \pm SD for continuous variables or as a percentage for categorical variable. For all analyses, a twosided probability $<0.05$ was considered to be significant. Univariable unpaired t-tests were used to compare age and TST diameter between HCWs who accepted therapy and those who did not. A $\mathrm{X}^{2}$ test was used to compare categorical variables between the groups. Variables associated with HCWs' perception of risk (eg, knowledge of risk magnitude, discussion with their caregiver, exposure to patient with $\mathrm{TB}$, travel to TB endemic area and receipt of BCG) were also compared between the two groups using a $\mathrm{X}^{2}$ test. In order to identify independent predictors for treatment non-acceptance, those variables which were significantly associated with treatment acceptance were then entered as independent variables to a logistic regression model with treatment acceptance as the dependent variable, adjusting for HCWs' age, occupation, gender and years at work. HCWs who did not accept treatment due to lack of medical recommendation were omitted from the model. $\mathrm{P}$ value of $\leq 0.05$ was required for entry into the model. Results are expressed as OR with 95\% CI.

\section{RESULTS}

Between 1 January 2000 and 31 December 2015, 219 HCWs were diagnosed with LTBI. Seventy-two (33\%) either refused to participate, or chose not to answer the questionnaire (online supplemental material 1). Overall, $147(67 \%)$ employees agreed to participate and answer the questionnaire. HCWs included physicians, nurses, therapists, laboratory workers, clerical staff and housekeeping, and had a similar distribution among participants and non-participants (data not shown).

Demographic characteristics of HCWs are shown in table 1 . The mean age was 43 years (range: 22-66years), median employment time was 6 years and mean follow-up time after the positive TST was 6.5 years (range: 2-18years). The majority of HCWs were women, and approximately half of HCWs received BCG in the past. Mean TST diameter was $15 \mathrm{~mm}$. Acceptance rate for LTBI treatment was $16 \%$. The overall completion rate among those who initiated therapy was $87 \%$ (20 of 23 HCWs). 
Table 1 Demographic and clinical characteristics of healthcare workers diagnosed with latent tuberculosis infection (LTBI)

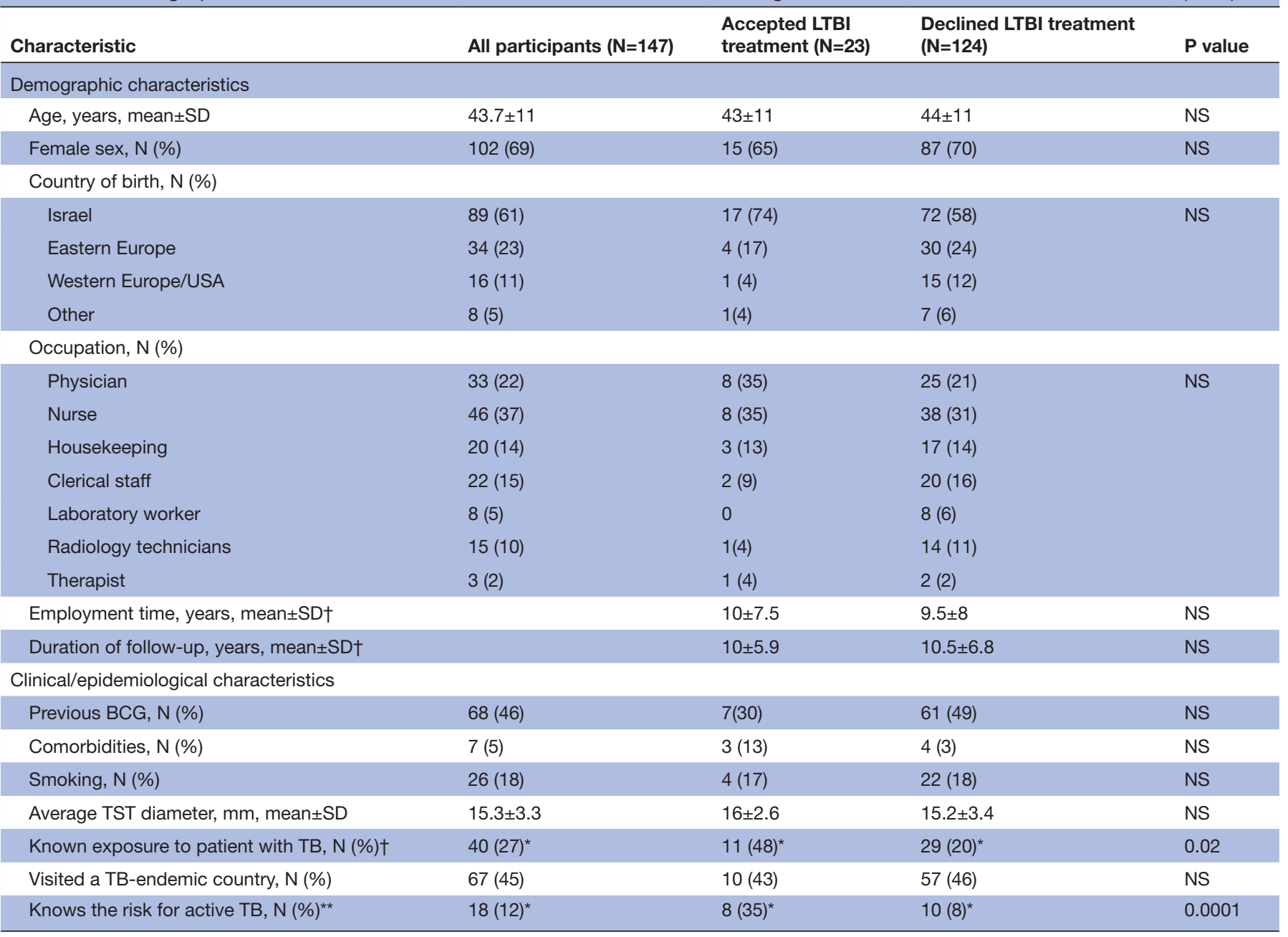

${ }^{\star} \mathrm{p}=0.02 ;{ }^{\star *} \mathrm{p}=0.0001$

†Employment time was calculated from start of employment to date of PPD. Follow-up time was calculated from date of PPD to date of interview.

$\mathrm{N}$, number of subjects; NS, not significant; PPD, purified protein derivative; TB, tuberculosis; TST, tuberculin skin testing.

\section{Reasons for non-acceptance}

Figure 1 shows the most common reasons for not accepting LTBI treatment. Fifty-two per cent of HCWs (64 of 124) reported that they did not accept LTBI treatment since they did not recall receiving a clear medical

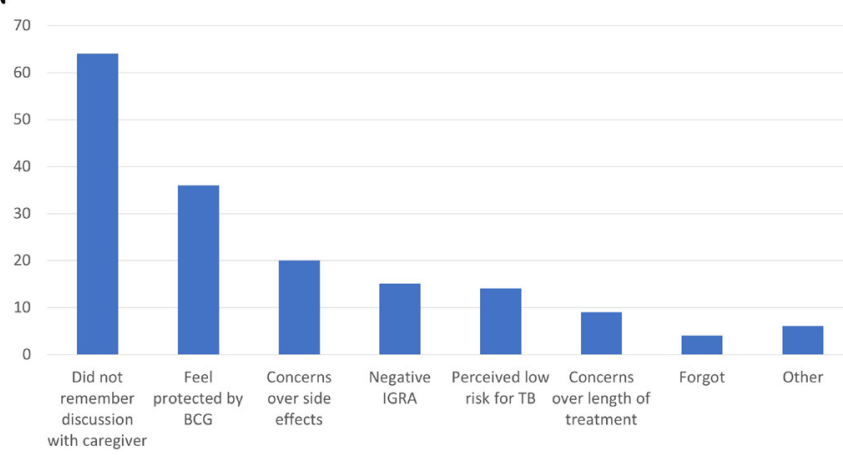

Figure 1 Reasons for non-acceptance of latent tuberculosis (TB) infection treatment. IGRA, interferon gamma release assay. recommendation. Of these, 34 did not recall why treatment was not recommended, 10 thought the reason was a PPD interpreted as borderline or false positive, and 4 said they were offered chest X-ray follow-up instead of LTBI treatment. The rest reported no recommendation due to pregnancy, interactions with other medications, TB treatment in childhood or because they do not have contact with patients. Thirty-six of HCWs did not accept treatment because they felt protected by previous BCG immunisation, 29 were concerned over side effects or length of treatment and 29 did not accept treatment due to negative IGRA or that they perceived their TB risk to be low.

\section{Predictors for non-acceptance}

Among HCWs who received medical recommendation for LTBI treatment, only 28\% (23 of 83) indeed accepted treatment. Fifty-two HCWs (35\%) recalled a discussion with their caregiver regarding the importance of therapy. This recall of the discussion had the strongest association with treatment acceptance: 23 of 52 HCWs (44\%) 


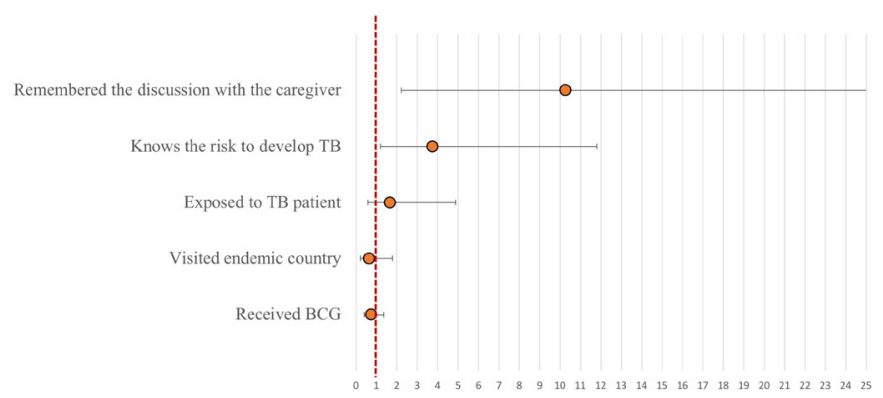

Figure 2 Results of logistic regression model: each circle represents the result of a regression model with acceptance of latent tuberculosis (TB) treatment as the dependent variable and the clinical characteristic as the independent variable. Each model was further adjusted for healthcare workers' age, occupation, gender and years at work. Circle indicates OR, vertical lines indicate $95 \%$ Cls and red line indicates OR reference of 1.

who remembered having a discussion with their caregiver regarding the importance of LTBI treatment actually received it (adjusted OR=10.2, 95\% CI: 2.2 to 47.6 , $\mathrm{p}=0.003$; figure 2). Knowing the risk of developing ТВ was associated with 3.7 increased odds to accept treatment (95\% CI: 1.2 to $11.8, \mathrm{p}=0.02)$. Other factors such as known exposure to a patient with active TB, travel to an endemic country or receipt of BCG were not significantly associated with treatment acceptance (figure 2).

\section{Completion rate and side effects}

Three patients stopped after initially accepting treatment. One because of elevated aminotransferases. Another four HCWs had elevated liver function tests, 5 of 23 (21\%).

\section{DISCUSSION}

In this study, we report the acceptance and completion rates of LTBI treatment among HCWs in a single institution and analyse the reasons for declining treatment. Only $16 \%$ of employees, who were diagnosed with LTBI and enrolled in the survey, accepted treatment. This rate is considerably lower than in previous reports-48\%$74 \% .{ }^{10}$ Recently, a study in the mid-western USA found an acceptance rate of $41 \%$ and a completion rate of $29 \%$ for LTBI treatment, with occupational and cultural factors affecting these rates. ${ }^{11}$

Medical recommendation that was perceived by HCWs as ambiguous was the main reason for not accepting LTBI treatment, followed by previous BCG vaccination, which HCWs perceived as either protective against TB or as a cause for false TST positive results. These two factors contributed to non-acceptance among 102 of 123 (82\%) HCWs who did not receive treatment. Concern of side effects was the third reason for declining LTBI treatment. The fourth reason for not accepting treatment was a negative IGRA test. In these cases, the provider may consider the TST as a false positive and therefore not recommend treatment.
HCWs' recall of discussing the importance of LTBI treatment with their caregiver increased the odds of accepting treatment by more than 10 -fold. Knowing the risk of reactivation of LTBI to active disease increased treatment acceptance odds by more than threefold. Positive TST following exposure to a patient with active TB was not significantly associated with treatment acceptance after adjusting for other factors. However, in their cohort, Pease et al found this to be significant in a multivariable analysis. ${ }^{12}$ National and international guidelines unequivocally recommend LTBI treatment, however, each caregiver may recommend the treatment more or less strongly. Treatment for LTBI is not mandatory and is not supervised for HCWs (eg, direct observed therapy) and this may further contribute to the low acceptance.

Contrary to the very low acceptance rate, the completion rate of LTBI treatment among those HCWs who accepted treatment was high-87\%. In comparison, a large study conducted in 32 designated clinics across the USA and Canada found the completion rate to be only $48 \% .^{5}$ This wide gap between the acceptance and completion rates in our population strengthens our assumption that HCWs who accepted treatment are those who were knowledgeable about the risk of active TB and regarding the benefits of LTBI treatment, thereby having high adherence to therapy.

The 2005 CDC guidelines for preventing Mycobacterium tuberculosis transmission in healthcare settings recommended annual testing for HCWs. ${ }^{13}$ Only very recently, in 2019, these recommendations were revised and no longer recommend routine serial screening of HCWs for LTBI, in the absence of a known exposure or ongoing transmission. ${ }^{9}$ The reasons for this revision were the facts that: (1) TB rates in the USA are steadily decreasing, ${ }^{14}$ (2) TB incidence rates among HCWs were similar to those in the general population ${ }^{15}$ and (3) the extremely low rate of TST conversion $(0.3 \%)$ among HCWs in a low TB incidence setting such as in the USA,with only a limited proportion attributable to occupational exposure. ${ }^{16}$ Moreover, in this recent work, none of the 123 TST converters developed active disease, despite the fact that less than half of them accepted LTBI treatment. Importantly, the 2019 guidelines emphasise the need for annual TB education to all healthcare personnel. ${ }^{9}$

Our study has several limitations. First, it relied on selfreport of HCWs. Self-report is prone to various kinds of bias, which may also be non-random. Nevertheless, the self-reporting process provides characterisation of HCWs' perceptions and beliefs regarding $\mathrm{TB}$, which is important in understanding how to improve adherence. Another major limitation is that details regarding the actual discussion between the healthcare provider and HCWs (eg, medical notes) were not available. Prospective monitoring of this discussion, as well as interviews of healthcare providers regarding their attitudes and practices, may provide opportunities to improve the TB screening and treatment process. 
Third, the participation rate in the study was only $67 \%$. The group of HCWs who participated in the study and those who chose not to participate were similar regarding their occupation, however other characteristics were not available for comparison. Finally, the time from TST conversion to enrolment was variable (2-18 years), which might have introduced a recall bias. There is also a concern that the recall bias may be differential (eg, those who accepted LTBI treatment may have a better recall of the discussion or a tighter follow-up).

In summary, we found that LTBI treatment acceptance among our HCWs is low. Our results suggest that focusing on educating HCWs regarding TB risk and the rationale for LTBI treatment is probably the key step that could increase the rates of LTBI treatment acceptance. The quality of the discussion between caregivers and HCWs was never surveyed or monitored in our institution although it seems to be associated with treatment acceptance.

Acknowledgements The authors thank Dr Leon Joseph for the critical review of this manuscript.

Contributors YW-W and MB-M have conceptualised and supervised the study. MB-M analysed the data and wrote the first draft. AR participated in data collection and critically read the manuscript. GP collected and analysed the data. All authors read the final draft and approved it.

Funding The authors have not declared a specific grant for this research from any funding agency in the public, commercial or not-for-profit sectors.

Competing interests None declared.

Patient consent for publication Not required.

Ethics approval The study was reviewed and approved by the Institutional Review Board at SZMC.

Provenance and peer review Not commissioned; externally peer reviewed.

Data availability statement Data are available upon reasonable request. Deidentified data of this study will be available upon request, through the Helsinki committee coordinator: leoras@szmc.org.il.

Supplemental material This content has been supplied by the author(s). It has not been vetted by BMJ Publishing Group Limited (BMJ) and may not have been peer-reviewed. Any opinions or recommendations discussed are solely those of the author(s) and are not endorsed by BMJ. BMJ disclaims all liability and responsibility arising from any reliance placed on the content. Where the content includes any translated material, BMJ does not warrant the accuracy and reliability of the translations (including but not limited to local regulations, clinical guidelines, terminology, drug names and drug dosages), and is not responsible for any error and/or omissions arising from translation and adaptation or otherwise.
Open access This is an open access article distributed in accordance with the Creative Commons Attribution Non Commercial (CC BY-NC 4.0) license, which permits others to distribute, remix, adapt, build upon this work non-commercially, and license their derivative works on different terms, provided the original work is properly cited, appropriate credit is given, any changes made indicated, and the use is non-commercial. See: http://creativecommons.org/licenses/by-nc/4.0/.

ORCID iD

Maskit Bar-Meir http://orcid.org/0000-0003-1616-2111

\section{REFERENCES}

1 Longo DFA, Kasper D, Hauser S. Harrison's principles of internal medicine. 18th edn. McGraw-Hill professional, 2011: 1340-59.

2 Trends in tuberculosis, 2018. Available: http://www.cdc.gov/tb/ publications/factsheets/statistics/tbtrends.html

3 Health IMO. Available: https://www.health.gov.il/publicationsfiles/tb_ birth_1989-2014.pdf

4 Getahun H, Matteelli A, Abubakar I, et al. Management of latent mycobacterium tuberculosis infection: who guidelines for low tuberculosis burden countries. Eur Respir J 2015;46:1563-76.

5 Horsburgh CR, Goldberg S, Bethel J, et al. Latent TB infection treatment acceptance and completion in the United States and Canada. Chest 2010;137:401-9.

6 Arguello Perez E, Seo SK, Schneider WJ, et al. Management of latent tuberculosis infection among healthcare workers: 10-year experience at a single center. Clin Infect Dis 2017;65:2105-11.

7 Sterling TR, Njie G, Zenner D, et al. Guidelines for the treatment of latent tuberculosis infection: recommendations from the National tuberculosis controllers association and CDC, 2020. MMWR Recomm Rep 2020;69:1-11.

8 Baussano I, Nunn P, Williams B, et al. Tuberculosis among health care workers. Emerg Infect Dis 2011;17:488-94.

9 Sosa LE, Njie GJ, Lobato MN, et al. Tuberculosis screening, testing, and treatment of U.S. health care personnel: recommendations from the National tuberculosis controllers association and CDC, 2019. MMWR Morb Mortal Wkly Rep 2019;68:439-43.

10 Camins BC, Bock N, Watkins DL, et al. Acceptance of isoniazid preventive therapy by health care workers after tuberculin skin test conversion. JAMA 1996;275:1013-5.

11 Swift MD, Molella RG, Vaughn AIS, et al. Determinants of latent tuberculosis treatment acceptance and completion in healthcare personnel. Clin Infect Dis 2020;71:284-90.

12 Pease C, Zwerling A, Mallick R, et al. The latent tuberculosis infection cascade of care in lqaluit, Nunavut, 2012-2016. BMC Infect Dis 2019;19:890.

13 Jensen PA, Lambert LA, lademarco MF, et al. Guidelines for preventing the transmission of Mycobacterium tuberculosis in healthcare settings, 2005. MMWR Recomm Rep 2005;54:1-141.

14 Stewart RJ, Tsang CA, Pratt RH, et al. Tuberculosis - United States, 2017. MMWR Morb Mortal Wkly Rep 2018;67:317-23.

15 Lambert LA, Pratt RH, Armstrong LR, et al. Tuberculosis among healthcare workers, United States, 1995-2007. Infect Control Hosp Epidemiol 2012;33:1126-31.

16 Dobler CC, Farah WH, Alsawas M, et al. Tuberculin skin test conversions and occupational exposure risk in US healthcare workers. Clin Infect Dis 2018;66:706-11. 